\title{
Comparison of Measurements from the Heidelberg Edge Perimetry with Standard Automated Perimeter in Glaucoma Patients
}

\author{
HANY A. KHAIRY, M.D.*; MARWA A. ZAKY, M.D.* and ABD EL-RAHMAN A. ISMAEL, M.Sc.** \\ The Department of Ophthalmology, Faculty of Medicine, Menofuia University* and The Department of Clinical Ophthalmology, \\ Ministry of Health, Imbaba Ophthalmic Hospital. Giza**, Egypt
}

\begin{abstract}
Background: Testing of the visual field remains the primary basis for evaluating glaucomatous visual function. Recently there have been innovations for perimetry that enhance its clinical utility the visual field was first measured by Thomas Young, in 1825, the outer aspect extends approximately $50^{\circ}$ superiorly, $60^{\circ}$ nasally, $70^{\circ}$ inferiorly and $90^{\circ}$ temporally.
\end{abstract}

Aim of Study: To compare visual field examination parameters, Mean Deviation (MD), Pattern Standard Deviation (PSD) in glaucoma patients performed on two different devices: Heidelberg Edge Perimeter (HEP) and Humphrey Visual Field analyser (HVF)

Patients and Methods: This is a randomized prospective, comparative, study conducted on thirty four eyes diagnosed as primary open angle glaucoma, those patients were recruited from the ophthalmic outpatient clinics at Rod-elfarag ophthalmic hospital in Cairo \& Imbaba Ophthalmic Hospital in Giza at time between May 2016 to June 2017. They had reliable visual field test and controlled by anti-glaucoma treatment without any other cause of secondary increased Intra Ocular Pressure (IOP), any surgical or laser intervention.

Results: There were a total of thirty four eyes where visual field examination by Heidelberg Edge Perimeter (HEP) and Humphrey Visual Field analyser (HVF) revealed no statistically significant difference between two perimeters except in severe glaucoma in which HVF showed more increase in PSD than HEP.

Conclusion: HVF tend to presents an increase in the level of PSD in severe glaucomatous defect than those measured by HEP.

Key Words: Glaucoma - Heidelberg edge perimeter - Perimetry - Visual field.

\section{Introduction}

GLAUCOMA is the second leading cause of blindness worldwide. The frequency of bilateral blindness amongst persons with glaucoma varies across

Correspondence to: Dr. Abd El-Rahman A. Ismael, E-Mail: Abdelrahman-abdelhay@hotmail.com populations, with substantial bilateral blindness from glaucoma observed in developing countries with poor access to eye care [1]. The most frequent Standard Automated Perimeter (SAP) is Humphrey, recommended by the European Glaucoma Society as the most useful for routine glaucoma diagnostics [2]. (SAP) or white on white $(\mathrm{w} / \mathrm{w})$ perimetry is best suited for patients with moderate to severe progressive disease and thereby offers continuity of care. The HEP monitor based system offers full dynamic range perimetry and has proven to deliver equivalent results to a projection type perimeter [3]. Another perimeter type is flicker defined perimetry which uses static perimeters using a different kind of stimulation-a flickering marker of periodically changing illumination [4]. The HEP offers a unique W/W perimetry using a Goldmann size III target for the $40 \mathrm{~dB}$ to $16 \mathrm{~dB}$ range in normal Visual Field (VF), from $15 \mathrm{~dB}$ to $0 \mathrm{~dB}$ range in low sensitivity (VF). The stimulus size is increased following the Goldmann equation to give perceptual equivalence. This unique approach enables full range Standard Automated Perimetry and ensures good test retest characteristics [5]. The aim of the study was to compare visual field examination parameters (MD, PSD) in glaucoma patients performed on two different devices: Heidelberg Edge Perimeter (HEP) and Humphrey Visual Field analyser (HVF).

\section{Patients and Methods}

This study was conducted on thirty four eyes diagnosed as Primary Open Angle Glaucoma (PO$A G)$ were defined as having an open anterior chamber angle on gonioscopy, those patients were recruited from the Ophthalmic Outpatient Clinics at Rod-El-Farag Ophthalmic Hospital in Cairo \& Imbaba Ophthalmic Hospital in Giza at time between May 2016 to June 2017, after signing informed consent about the study procedure and 
approval of Ethical Committee. They had a reliable visual field test that showed significant changes and an Optic Nerve Head (ONH) showing glaucomatous disc changes, without other cause of secondary increased Intra Ocular Pressure (IOP) or any retinal disease or surgical or laser intervention, IOP was controlled by anti-glaucoma treatment, (average intraocular pressure $16.3 \mathrm{mmHg}$ ). Diabetics and patients with neurological medical history were not qualified. Complete medical, ocular and family history was taken. Refractive error and best corrected visual acuity were measured and a basic ophthalmologic examination including anterior segment examination by the slit lamp (Topcon Corp, Tokyo, Japan) to examine the cornea to determine if its clarity will permit proper evaluation of the posterior segment. The pupil was examined in the un-dilated condition and its reaction to light was assessed. The anterior segment and the iris were examined and any possible causes of secondary elevation of IOP were assessed. The Intraocular Pressure (IOP) was measured using (Goldmann applanation tonometer, Tokyo, Japan) after instillation of topical anesthetic drops and staining the precorneal tear film with fluorescein stain. The pupil was then dilated using tropicamide $1 \%$ eye drops and phenylephrine $2.5 \%$ eye drops. The lens was then examined for presence of media opacity like cataract. A Gonioscopy was performed for all patients. Angle was graded according to Shaffer system: Grade IV: 40 degrees: Ciliary band visible. Grade III: 30 degrees: Scleral spur visible. Grade II: 20 degrees: Anterior trabecular meshwork visible. Grade I: 10 degrees: Schwalbe's line visible. Grade 0: Closed: Schwalbe's line not visible. Visual field was performed for all patients. Visual field test was performed for all patients using Humphrey perimeter 740 Visual Field Analyser \& Heidelberg Edge Perimeter (HEP). We used HEP software release 1.9.10.0, which includes HEP Acquisition Module (AQM) v. 1.9.10.0, HEP Viewing Module (VWM) v. 1.9.10.0. All tests were performed using SITA standard strategy on (HVF) (Humphrey visual field, Carl Zeiss Meditec, Jena, Germany) and ASTA standard Strategy on (HEP) (Heidelberg Edge Perimeter, Heidelberg Engineering, Heidelberg, Germany) using SAP III stimulus ( 0.43 degree and background luminance was $10 \mathrm{~cd} / \mathrm{m}^{2}$ ) on both devices. Stimulus duration was $200 \mathrm{msec}$ with interval time $600 \mathrm{msec}$. Because a scale in HEP runs from 0 to $25 \mathrm{~dB}$, it cannot be easily compared with the results obtained using the Humphrey perimeter, for this reason, HEP values were converted into a scale generally assumed for SAP perimeters (including Humphrey's), which allowed for a comparison of the obtained results. Visual field was defined abnormal according to the Anderson's criteria (Glaucoma hemi field test outside the normal limits and more than or equal three adjacent points that have a probability less than $5 \%$, one of which must have a probability less than $1 \%)$. Eyes were classified according to severity of glaucoma to mild glaucoma which range from $(-2.0:-6.0)$, moderate $(-6.1:-12.0)$ and severe $>-12.01$ according to Humphrey visual field results. Comparison between each category was done separately. Severity distribution among the studied eyes were mild $35.3 \%$ (12/34), moderate $41.2 \%$ (18/34) and sever $23.5 \%$ (4/34) data were collected with the formal patient consent after approval of ethical committee and compared visual field results performed by the perimetrist on both perimeters.

\section{Statistical analysis:}

Data were collected, revised, coded and entered to the Statistical Package for Social Science (IBM SPSS, Inc. IBM, Chicago, USA) Version 23. The quantitative data were presented as mean, standard deviations and ranges when their distribution found parametric and median with Inter-Quartile Range (IQR) when non parametric. Also qualitative variables were presented as number and percentages. The comparison between two paired groups with quantitative data and parametric distribution were done by using Paired $t$-test while the comparison between two paired groups with quantitative data and non-parametric distribution were done by using Wilcoxon Rank test. Spearman correlation coefficients were used to assess the correlation between two quantitative parameters in the same group. The confidence interval was set to $95 \%$ and the margin of error accepted was set to 5\%. So, the $p$ value was considered significant as the following: $p<0.05$ : Significant.

\section{Results}

The study included thirty four eyes (as selected inclusion criteria) of normal healthy individuals seeking visual field examination. The pre-examination characteristics of both groups showed no significant statistical difference between them as regards age, all of these patients were diagnosed as POAG, patients eyes were on medical treatment; six $(6 / 34)$ on combined carbonic anhydrase inhibitor and B blocker, six (6/34) on B blockers, fourteen (14/34) on prostaglandins analogues, and eight (8/34) on other anti-glaucoma drops. All visual field testing were reliable in all eyes with a mean test duration of 11:09min \pm 2:07 ranging from 7:09 to $15: 48$ minutes. With glaucoma hemi field test was outside normal limits in all patients. Mean 
Deviation (MD) was calculated separately for each eye right and left, first on Humphrey Visual Field (HVF) and second on Heidelberg Edge Perimeter (HEP), the measured MD values on both devices showed no significant statistical differences between them as regards $(\mathrm{HVF}=-6.73, \mathrm{HEP}=-7.66)$, a Pattern Standard Deviation (PSD) value was calculated separately for each eye right and left first on HVF and second on HEP, the measured PSD values on both devices showed no significant statistical differences between them as regard $(\mathrm{HVF}=5.07, \mathrm{HEP}=4.42)$ (Table 1). Eyes were classified according to severity of glaucoma to mild glaucoma which range from $(2.0: 6.0)$, moderate $(-6.1:-12.0)$ and severe $>-12.01$ according to Humphrey visual field results, severity distribution among the studied eyes were mild $35.3 \%(12 / 34)$, moderate $41.2 \%(18 / 34)$ and severe $23.5 \%(4 / 34)$. A comparison of MD by HVF and HEP in mild glaucoma was $-3.89,-3.75$ respectively, and for moderate glaucoma was $-7.95,-7.48$ respectively and for severe glaucoma was $-22.2,-22.87$ respectively which showed no statistically significant difference found between HVF and HEP regarding
MD in mild, moderate and severe case (Table 2). A comparison of PSD by HVF and HEP in mild glaucoma was 2.67, 3.09 respectively, and for moderate glaucoma was $4.78,4.51$ respectively and for severe glaucoma was $9.17,6.25$ respectively which shows no statistically significant difference found between HVF and HEP regarding PSD in mild, moderate but there was statistically significant increase in the level of PSD in severe cases measured by HVF than those measured by HEP Fig. (1). A comparison between HVF and HEP regarding MD and PSD in mild, moderate and severe eyes separately revealed that there was no statistically significant difference found between HVF and HEP regarding MD in mild, moderate and severe cases with $p$-value $=0.286,0.865$ and 0.327 respectively. While there was no statistically significant difference found between HVF and HEP regarding PSD in mild and moderate cases with $p$-value= 0.500 and 0.695 respectively but, also there was statistically significant increase in the level of PSD in severe cases measured by HVF than those measured by HEP with $p$-value $=0.013$.

Table (1): Comparison between Humphrey Visual Field (HVF) and Heidelberg Edge Perimeter (HEP) regarding Mean Deviation (MD) and Pattern Standard Deviation (PSD) in all the studied eyes.

\begin{tabular}{|c|c|c|c|c|}
\hline & HVF & HEP & Test value & $p$-value \\
\hline \multicolumn{5}{|l|}{$M D:$} \\
\hline Median (IQR) & $-6.73(-10.63--4.28)$ & $-7.66(-16.35--3.73)$ & $-0.282 *$ & 0.778 \\
\hline Range & $-32.71--1.9$ & $-28.52--2.01$ & & \\
\hline \multicolumn{5}{|l|}{ PSD: } \\
\hline Mean \pm SD & $5.07 \pm 3.48$ & $4.42 \pm 2.60$ & $1.436^{\bullet}$ & 0.160 \\
\hline Range & $1.62-15.32$ & $1.36-10.65$ & & \\
\hline
\end{tabular}

*: Wilcoxon Rank test.

-: Paired $t$-test.

Table (2): Comparison between Humphrey Visual Field (HVF) and Heidelberg Edge Perimeter (HEP) regarding Mean Deviation (MD) and Pattern Standard Deviation (PSD) in mild, moderate and severe eyes separately.

\begin{tabular}{lllll}
\hline & \multicolumn{1}{c}{ HVF } & \multicolumn{1}{c}{ HEP } & Test value & $p$-value \\
\hline Mild: & & & \\
MD (median (IQR)) & $-3.89(-4.28--2.99)$ & $-3.75(-5.88--3.03)$ & $-1.067^{*}$ & 0.286 \\
PSD (mean \pm SD) & $2.67 \pm 1.49$ & $3.09 \pm 1.25$ & $0.698 \bullet$ & 0.500 \\
Moderate: & & & & \\
MD (median (IQR)) & $-7.95(-9.38--6.32)$ & $-7.48(-13.98--3.23)$ & $-0.170^{*}$ & 0.865 \\
PSD (mean \pm SD) & $4.78 \pm 2.33$ & $4.51 \pm 2.1$ & $0.401 \bullet$ & 0.695 \\
Severe: & & & & \\
MD (median (IQR)) & $-22.2(-27.15--18.43)$ & $-22.87(-27.78--15.94)$ & $-0.980^{*}$ & 0.327 \\
PSD (mean \pm SD) & $9.17 \pm 3.77$ & $6.25 \pm 3.79$ & $3.303 \bullet$ & 0.013 \\
\hline * : Wilcoxon Rank test. & HVF : Humphrey Visual Field. & \\
: Paired $t$-test. & MD : Mean Deviation. & \\
HEP : Heidelberg Edge Perimeter. & PSD : Pattern Standard Deviation. &
\end{tabular}




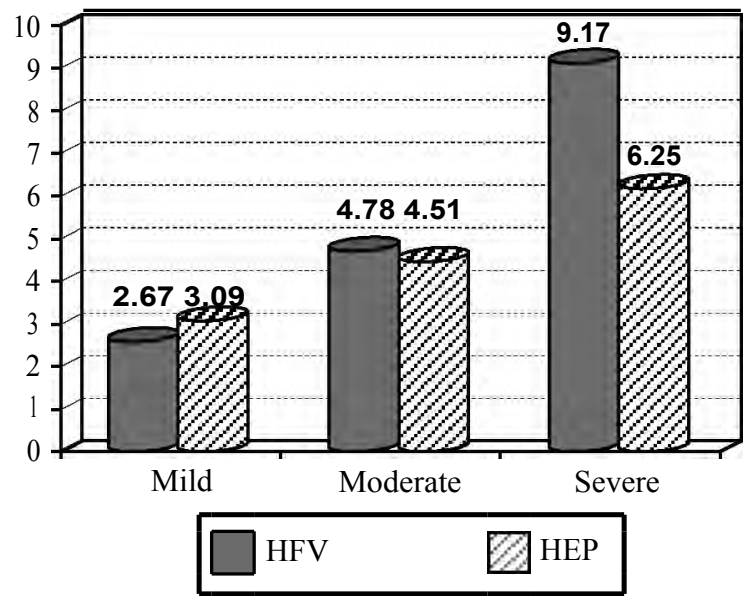

Fig. (1): Pattern standard deviation in mild, moderate and severe eyes by Humphrey visual field and Heidelberg edge perimeter.

\section{Discussion}

Glaucoma is the second leading cause of blindness in the world, the main goal of glaucoma management is to diagnose this disease when it is asymptomatic. Visual field testing is essential in the diagnosis and monitoring of glaucoma. However it is known that standard perimetry cannot detect VF defects until 40\%-50\% of ganglion cells have been lost, Quigley et al. [6] . In our study we tried to compare two types of perimetry using the same SAP III stimulus in both perimeter, the first was Heidelberg Edge Perimeter (HEP) and the second was Humphrey Visual Field analyser (HVF) for detecting glaucomatous field defect in a diagnosed POAG. Mean Deviation (MD) and Pattern Standard Deviation (PSD) were the main parameter in differentiating and detecting visual field changes. In our study, we found no statistically significant difference between HVF and HEP regarding MD in mild, moderate and severe cases with $p$-value $=$ $0.121,0.584$ and 0.494 respectively. While there was no statistically significant difference found between HVF and HEP regarding PSD in mild and moderate cases with $p$-value $=0.500$ and 0.695 respectively but, also there was statistically significant increase in the level of PSD in severe cases measured by HVF than those measured by HEP with $p$-value $=0.013$. This clarify that there was no statistically significant between two perimeters except in severe glaucoma in which HVF showed more increase in PSD than HEP than HEP. This study agrees with Ichhpujani et al., [7] in that HEP results have similarities to SAP results, HEP and HVF have significant correlation to each other. It also agrees with Hasler et al., [8] in that HEP seems to be more sensitive than conventional static perimetry in early detection of visual field alteration in patients between ocular hypertension and incipient glaucoma. This study agrees with Calvo Perez et al., [9] who reported that both perimetries showed a good correlation between their indices and global values, HEP tend to present MD and PSD values lower than Humphrey. It also disagrees with Kamil Kaczorowski et al., [10] who compared both perimeters but in patients with ocular hypertension, they found that MD results obtained with HEP are lower than MD obtained with Humphrey perimeter, which might mean that HEP yields more accurate results and detects very early losses in the visual field, but we found that HVF presents PSD more HEP only in severe cases. Measuring glaucoma progression using visual fields is very difficult, even with sophisticated progression software, since many of our glaucoma patients are poor visual field testers; subjective analysis of glaucoma progression in these patients is very difficult. The ability to detect early field changes could be a valuable tool in assessing the diagnosis and progression of glaucoma, our study revealed that both HEP \& HVF testing are useful methods in managing these patients. But the ability to measure early field defect is an important significant advantage for HEP over HVF in this group of patients. Nowadays it is said that glaucoma damage doesn't only affect optic nerve fibers, but also Retinal Ganglion Cells [RGC] Chen et al., [11]. The type and timing of cellular changes leading to RGC loss in glaucoma remain incompletely understood, including whether specific RGC subtypes are preferentially impacted at early stages of the disease Huberman et al., [12] It seems that magnocellular retinal cells (M-cells) are more sensitive than parvocellular retinal cells (P-cells) and become some of the first to be damaged in glaucoma [13]. Since these cells are very sensitive to glaucoma damage, they suffer complete loss at an early stage of glaucoma Hemmi et al., [14], which in turn influences the visual function and thus the visual field as well. Therefore, early detection of these defects is crucial to starting the treatment in time. It has been noted that substantial retinal ganglion cell damage can happen long before its detection using standard examinations assessing RGC damage Hood et al., [15]. Some authors, however, claim that M-cell damage does not affect the results of visual field examinations Swanson et al., [16].

\section{Conclusion:}

There was no statistically significant between two perimeters except in severe glaucoma in which HVF show more increase in PSD than HEP. 


\section{References}

1- RESNIKOFF S., PASCOLINI D., ETYA'ALE D., KOCUR I. and PARARAJASEGARAM R.: Global data on visual impairment in the year 2002: Programme for the Prevention of Blindness and Deafness: World Health Organization. WHO-Report; WHO 04-138, 2002.

2- HITCHINGS R.: Terminology and Guidelines for Glaucoma 2015. Available from: http://www.eugs.org/ eng/ EGS_guidelines.asp.

3- FERRERAS A., POLO V., LARROSA J.M., PABLO L.E., PAJARIN A.B., PUEYO V. and HONRUBIA F.M.: Can frequency-doubling technology and short-wavelength automated perimetries detect visual field defects before standard automated perimetry in patients with preperimetric glaucoma? J. Glaucoma, 16 (4): 372-83, 2007.

4- SCHIEFER U., PATZOLD J., WABBELS B. and DANNHEIM F.: Conventional techniques of visual field examination: Part 4 Static perimetry: Interpretationperimetric indices-follow-up-perimetry in childhood. Ophthalmology, 103: 235-54, 2006.

5- Heidelberg Engineering GmbH 93 228-004.E11@ 2011.

6- QUIGLEY H.A., ENGER C., KATZ J., SOMMER A., SCOTT R. and GILBERT D.: Risk factors for the development of glaucomatous visual field loss in ocular hypertension. Arch. Ophthalmol., 112 (5): 644-9, 1994.

7- P. ICHHPUJANI, L. KATZ, D.C. LO, A. CHOKSI, J. ROME, J.S. MYERS and G.L. SPAETH: Structure and Function Correlation Using Heidelberg Edge Perimeter, Humphrey Visual Field and Heidelberg Retinal Tomography 3: Investigative Ophthalmology. Visual Science, Vol. 51: 4917, 2010.

8- HASLER S. and STÜRMER J.: First experience with the Heidelberg Edge Perimeter on patients with ocular hypertension and preperimetric glaucoma: Augenklinik, kantonsspital Winterthur, schweiz. Simon. Kiln monbl augenheilkd, 229 (4): 319-22, 2012.
9- P. CALVO PEREZ, L. GIL-ARRIBAS, A. FERRERAS, S. OTIN, I. ALTEMIR, S. FERNANDEZ, L. PABLO JULVEZ and I. FUERTES: Relationship between flicker FDF perimetry and standard automated perimetry: 2010 European Association for Vision and Eye Research Conference Doi: 10.1111/j.1755-3768.2010.4354.x.

10- KAMIL KACZOROWSKI1, MALGORZATA MULAK, DOROTA SZUMNY, MARTA BARANOWSKA, JOANNA JAKUBASZKO-JABLOЙSKA and MARTA MISIUK-HOJLO: "Comparison of Visual Field Measurement with Heidelberg Edge Perimeter and Humphrey Visual Field Analyzer in Patients with Ocular Hypertension", 25: 937-44, 2016

11- CHEN Y.S., GREEN C.R., DANESH-MEYER H.V. and RUPENTHAL I.D.: Neuroprotection in the treatment of glaucoma-A focus on connexin43 gap junction channel blockers. Eur. J. Pharm. Bio. Pharm., 2015.

12- EL-DANAF R.N. and HUBERMAN A.D.: Characteristic patterns of dendritic remodeling in early-stage glaucoma: Evidence from genetically identified retinal ganglion cell types: J. Neurosc., 35: 2329-43, 2015.

13-KERRIGAN-BAUMRIND L.A., QUIGLEY H.A., PEASE M.E., KERRIGAN D.F. and MITCHELL R.S.: Number of ganglion cells in glaucoma eyes compared with threshold visual field tests in the same persons: Invest. Ophthalmol. Vis. Sci., 41: 741-8, 2000.

14- MADDESS T., HEMMI J.M. and JAMES A.C.: Evidence for spatial aliasing effects in the Y-like cells of the magnocellular visual pathway: Vision Res., 38: 1843-59, 1998.

15- HOOD D.C. and KARDON R.H.: A framework for comparing structural and functional measures of glaucomatous damage: Prog. Ret. Eye Res., 26: 688-710, 2007.

16- WHITE A.J., SUN H., SWANSON W.H. and LEE B.B.: An examination of physiological mechanisms underlying the frequency-doubling illusion: Invest. Ophthalmol. Vis. Sci., 43: 3590-9, 2002.

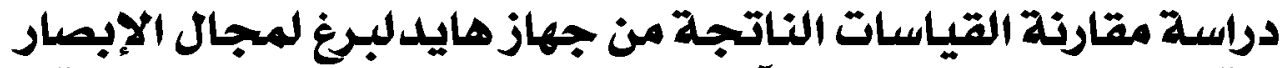

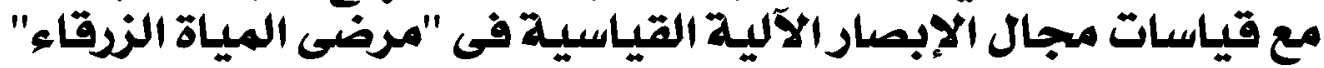

$$
\begin{aligned}
& \text { تعتبر المياه الزرقاء من الآمراض الصامتة التى تؤثر سلبا من قدرة العين على الإبصار ولهذا كان لهجال الإبصار دود كبير فى تشخيص } \\
& \text { ومن ثم علاج هذا المرض. المياه. }
\end{aligned}
$$

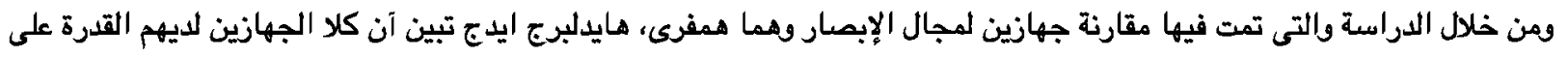

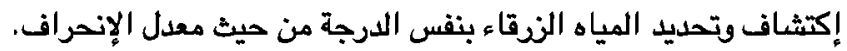

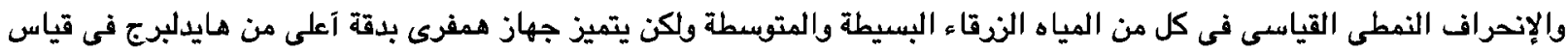

$$
\begin{aligned}
& \text { الإنحرافـ النمطى القياسى فى المياه الزّقاء الثـديدة. }
\end{aligned}
$$

